\title{
Crystalline boron nitride nanosheets by sonication-assisted hydrothermal exfoliation
}

\author{
Zhaobo TIAN, Kexin CHEN*, Siyuan SUN, Jie ZHANG, \\ Wei CUI, Zhipeng XIE, Guanghua LIU* \\ State Key Laboratory of New Ceramics and Fine Processing, School of Materials Science and \\ Engineering, Tsinghua University, Beijing 100084, China
}

Received: June 10, 2018; Revised: July 17, 2018; Accepted: July 25, 2018

(C) The Author(s) 2019.

\begin{abstract}
A simple method to prepare two-dimensional hexagonal boron nitride (h-BN) scalably is essential for practical applications. Despite intense research in this area, high-yield production of two-dimensional h-BN with large size and high crystallinity is still a key challenge. In the present work, we propose a simple exfoliation process for boron nitride nanosheets (BNNSs) with high crystallinity by sonication-assisted hydrothermal method, via the synergistic effect of the high pressure, and cavitation of the sonication. Compared with the method only by sonication, the sonication-assisted hydrothermal method can get the fewer-layer BNNSs with high crystallinity. Meanwhile, it can reach higher yield of nearly $1.68 \%$, as the hydrothermal method with the yield of only $0.12 \%$. The simple sonication-assisted hydrothermal method has potential applications in exfoliating other layered materials, thus opening new ways to produce other layered materials in high yield and high crystallinity.
\end{abstract}

Keywords: boron nitride nanosheet (BNNS); high crystallinity; sonication-assisted; hydrothermal method

\section{Introduction}

In recent years, the discovery of graphene, a one-atom thick flat allotrope of carbon, has inspired researchers to explore other two-dimensional materials for the unique properties and various promising applications [1-4]. Among the two-dimensional materials, atomically thin hexagonal boron nitride (h-BN), has attracted more attention due to the excellent chemical stability, outstanding mechanical and thermal properties [5], and

\footnotetext{
* Corresponding authors.

E-mail: K. Chen, kxchen@mail.tsinghua.edu.cn; G. Liu, liugh02@163.com
}

potential applications in ultraviolet-light emitters, high-temperature oxidation-resistant coatings, and an ideal substrate for high-quality graphene electronics [6-11]. All of these applications need an amount of highcrystallinity BNNSs. Although the BNNS is the structure analogue of graphene, some methods usually used for synthesizing graphene, such as the Hummer's and related graphene oxide methods, are not suitable for the preparation of BNNS, because of slightly ionic bonding between the BN layers (i.e., lip-lip interactions), which is stronger than the van der Waals' force between graphite layers.

With years' efforts, several modified methods for effectively fabricating few-layer BNNSs have been 
reported, including mechanical cleavage [12], liquid exfoliation $[13,14]$, and chemical vapor deposition $[15,16]$. However, there are still some limits for these modified methods. For example, the mechanical cleavage method using the shear force to exfoliate the BN powders can damage the structure of the obtained sheets, reducing crystallinity. Some researchers use specific solution as the medium to mill the $\mathrm{BN}$ powders to get well dispersed BNNSs, while bringing a little impurity into the production [12]. The chemical vapor deposition can get few or monolayer BNNS, with high crystallinity, except the high cost and high temperature. Recently, liquid-phase exfoliation of layered materials with the assist of sonication or hydrothermal treatment, has been found to have great potential for the production of layered materials [13]. Nevertheless, the traditional high-power sonicationassisted liquid exfoliation methods cannot get largelateral-size few-layered BNNSs and make damage on the nanosheets, due to the large energy of sonication, which limits its application [17]. The other liquid exfoliation method, hydrothermal treatment, always uses the molten hydroxides, introducing some impurity residue, and the yield is lower compared with the sonication. Compared the two methods, it is obvious that the sonication-assisted liquid exfoliation method can get higher yield while the crystallinity is lower and the size is smaller than the hydrothermal treatment method.

Herein, inspired by the characteristic and mechanism of the sonication-assisted liquid exfoliation and hydrothermal treatment method, we propose a simple method for exfoliating h-BN by using low-power sonication-assisted hydrothermal method. Under the pressure produced on the hydrothermal process, the solution can be immersed into the layers. Meanwhile, with the assist of energy of the sonication, the micrometer-sized bubbles or voids in liquids immersed into the layers can be formed and collapsed due to pressure fluctuation, thus exfoliating the bulk h-BN into nanosheets. The $60 \%$ tertiary butanol aqueous solution was used to exfoliate the $\mathrm{h}-\mathrm{BN}$ as the surface tension is similar with the BNNSs. Meanwhile, the solution was easy to remove, without leaving any residual impurity into the BNNSs. More importantly, the method results in defect-free single- or few-layer BNNSs with a yield of $1.68 \mathrm{wt} \%$ of the starting h-BN powders, and the yield could potentially be improved to $3 \mathrm{wt} \%$ by sediment recycling.

\section{Experimental procedure}

It should be pointed out that it is impossible to make sure every batch of commercially-obtained h-BN powders is identical. Notably, the chemistry properties of h-BN are very dependent on $\mathrm{BN}$ sample preparation, purity, crystallinity, and microstructure. Because of this, the results of the experiment may be different from batch to batch.

In order to get BNNSs in sonication-assisted hydrothermal conditions, the h-BN powders (Alfa Aesar Co.) and 60\% tertiary butanol solution (Beijing Chemical Co., Beijing, China) were fed into a poly (tetrafluoroethylene)-lined stainless steel. Then, the reactor was placed into the sonication cleaner with the rated power of $300 \mathrm{~W}$, and kept in the temperature of $355 \mathrm{~K}$ for $2 \mathrm{~h}$, as the schematic shown in Fig. 1. After sonication-assisted hydrothermal exfoliation, the dispersions were transferred into a tube and centrifuged at $3500 \mathrm{rpm}$ for $30 \mathrm{~min}$. Then, the top $90 \mathrm{~mL}$ supernatant (out of $100 \mathrm{~mL}$ ) was carefully collected to character the BNNSs, and the sediment could be cycled for further hydrothermal exfoliation. With the purpose of proving the valid of the method, the controlled trials were designed and conducted. In order to distinguish these methods, the h-BN powders treated by sonication were named as SC-BN, treated by hydrothermal named as HT-BN, and treated by sonication-assisted hydrothermal named as SH-BN, respectively.

The microstructure of raw $\mathrm{BN}$ powders and sediment of centrifugal treatment were observed using a scanning electron microscope (SEM; Melin, Zeiss Corporation, Germany) at an accelerating voltage of $5.0 \mathrm{kV}$. The morphology and the diffraction pattern of

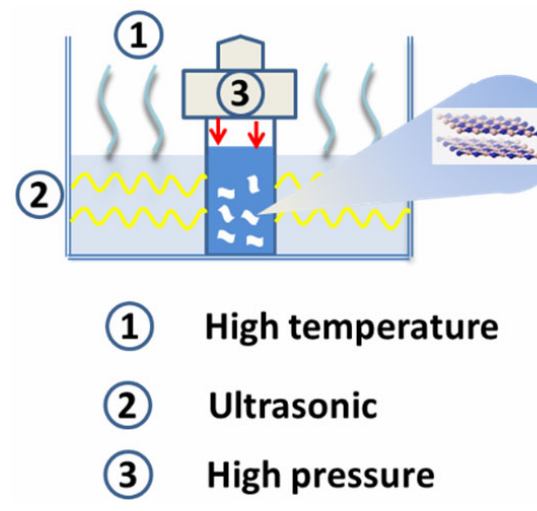

Fig. 1 Schematic of sonication-assisted hydrothermal method. 
BNNS were detected by high-resolution transmission electron microscope (TEM; JEM-2100F, JEOL, Japan), operated at an accelerating voltage of $200 \mathrm{kV}$. The TEM samples were prepared by drying a droplet of the BNNS suspension on a carbon grid. The thickness and the lateral size of the BNNS were analyzed by the atomic force microscopy (AFM) images obtained with a Nanoscope MultiMode instrument (Digital Instruments/ Bruker Systems), operated in the air tapping mode. The samples were prepared by depositing the BNNS suspension on a Si substrate and dried in a vacuum oven for $2 \mathrm{~h}$ before AFM measurement. Raman spectra were excited using the $633 \mathrm{~nm}$ excitation source from $\mathrm{He}-\mathrm{Ne}$ laser and collected by a micro-Raman spectrometer (HR800, HORIBA, France) at room temperature.

\section{Results and discussion}

Scanning electron microscopy (SEM) analyses (Fig. 2(a)) show that the starting powders consist of flakes with the lateral size of 2-4 micrometres and thickness of hundreds of nanometres. In comparison, the sediment separated by centrifugating the solution treated with sonication-assisted hydrothermal method, contains smaller flakes, with a lateral size of $<2 \mu \mathrm{m}$ (Fig. 2(b)). It is obvious that these flakes are consisted of many nanosheets stacked together loosely (Fig. 2(b)), compared with the pristine particles (Fig. 2(a)). Notably, the sonication-assisted hydrothermal method can overcome the intermolecular force effectively, to exfoliate the intact particle into flakes. Meanwhile, it is obvious that the sediment can be exfoliated into nanosheets furtherly to improve the yield. In addition, the method not only results in the BN particle reducting of thickness, but also the lateral size of the (002) BNNSs becomes smaller, compared with pristine particles. This is an inevitable phenomenon, as some researches have pointed out that the mechanical force can peel off
BNNS from some defective areas of precursor $\mathrm{BN}$ particles, meanwhile reducing the lateral size in some degree [6,18]. Eventhough, the lateral size is also larger than the traditional method using high-power sonication-assisted liquid exfoliation methods.

Transmission electron microscopy (TEM) was employed to count the thickness and further analyze the structures of the BNNS exfoliated by the sonicationassisted hydrothermal method. Some of the stacked BNNSs with single or a few layers in lager size can be observed (Fig. 3(a)). Due to ultimately thin shapes, the BNNSs were entirely transparent to an electron beam, making it possible to count the layers. As shown in Fig. 3 (b), it is obvious that the suspended films are consisted of the few-layer BNNSs (4 to 6 layers). The incident electron beam is along the [002] direction, thus perpendicular to the (002) crystal face. Through the electron diffraction pattern of the BNNS, the pattern reveals a typical six-fold symmetry natural of h-BN and demonstrates the well-crystallized structure of the BNNS. Meanwhile, as shown in Fig. 3(c), the nanosheet presents a well integrity atomic structure. Furthermore, according to the geometrical relationship, the spacing between the atoms is $1.43 \AA$, which is quite close to the well-known length of a B-N bond of $1.44 \AA$. All the evidences make it clear that the sonication-assisted hydrothermal method can get high-crystallinity BNNS with a few layers (4 to 6). Compared with the TEM images of SC-BNNS and HT-BNNS, shown in Figs. 4(a) and 4(b), the ST-BNNS appears the same high crystallinity with HT-BNNS, while the SC-BNNS is poor.

Atomic force microscopy (AFM) was also used to study the nanosheets' thickness and fine structure, which was unobservable in SEM and TEM clearly. Figure 5(a) shows a few flat nanosheets of ST-BNNS with thickness of about $2.4 \mathrm{~nm}$ (the height profile of Fig. 5(b)). A previous report shows that the AFM height of a monolayer BNNS increases from 0.33 to
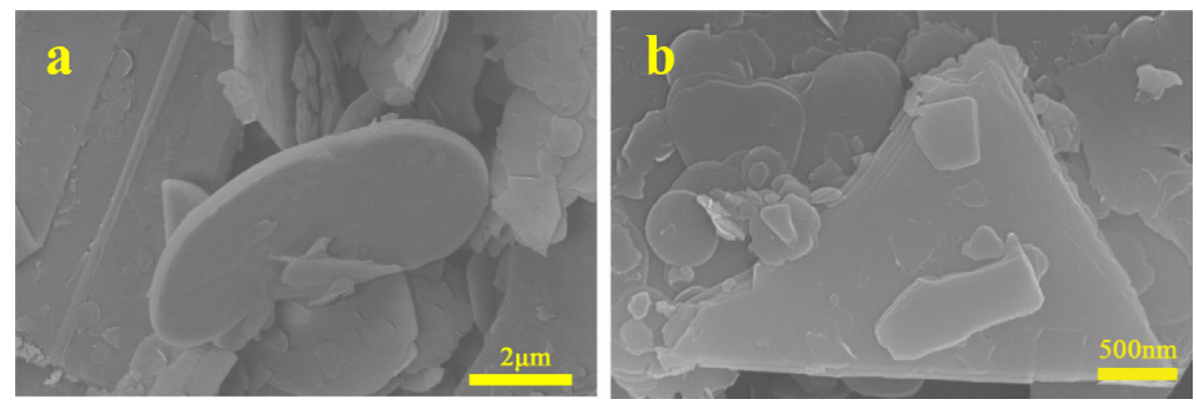

Fig. 2 SEM images of (a) the pristine h-BN powders and (b) the sediment of SH-BN after centrifugation. 

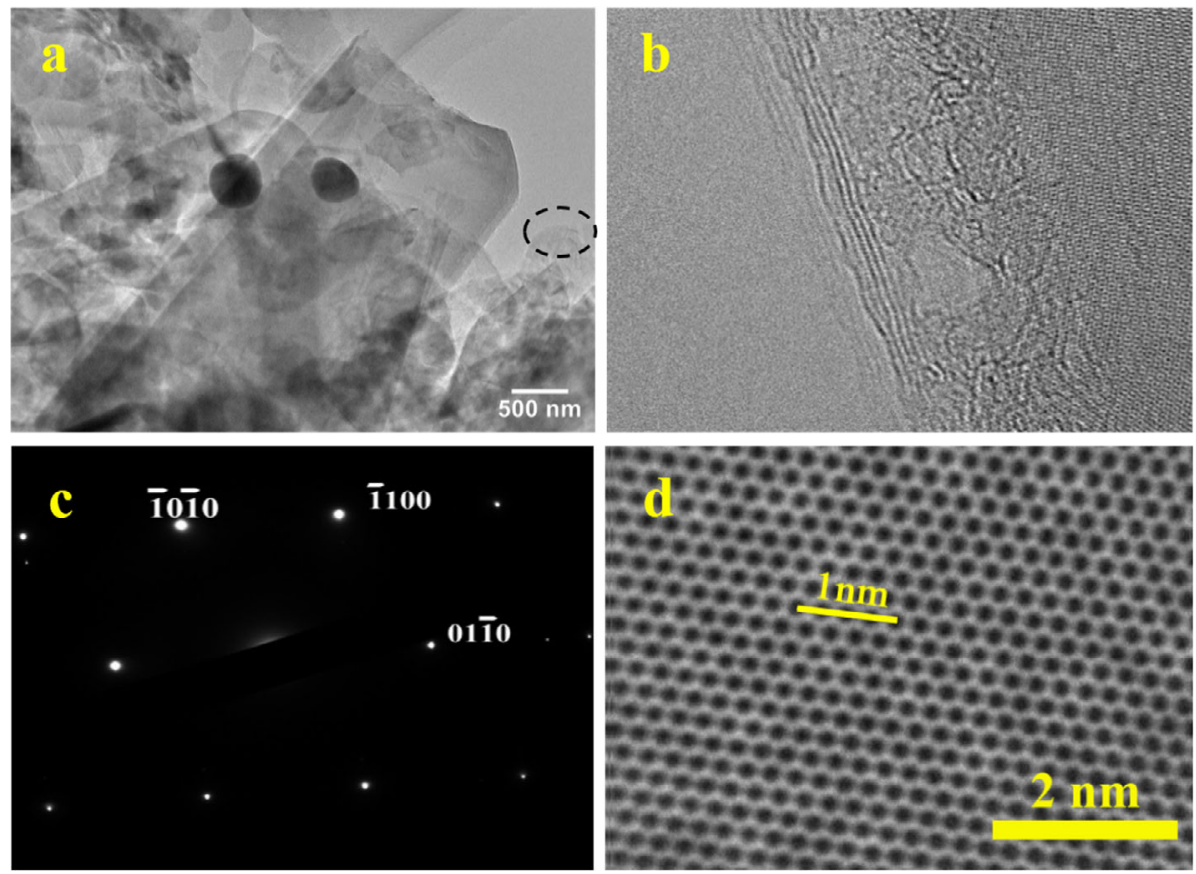

Fig. 3 (a) Low-magnification TEM image of BNNS. (b) High-resolution image of the encircled region in (a); (c) SAED of the region in (a); (d) HRTEM and structure model of BNNS.
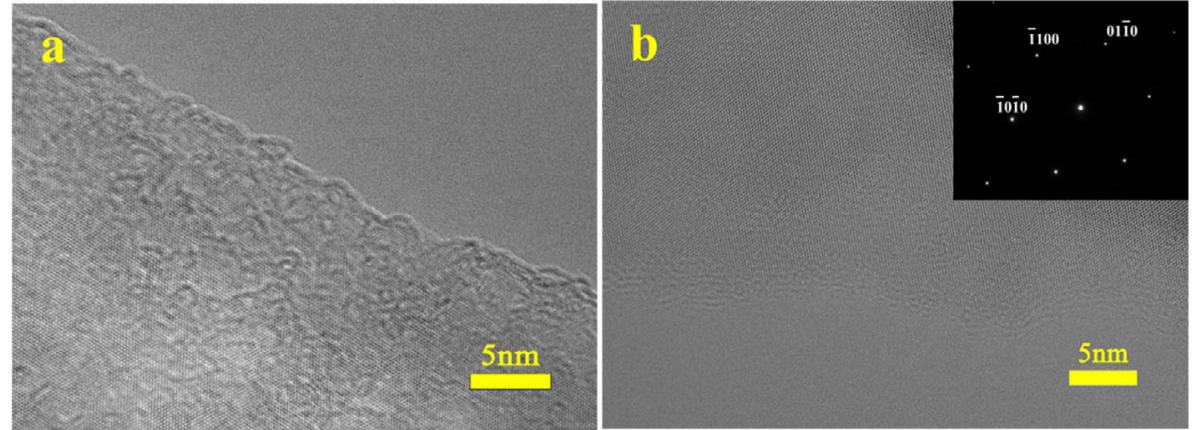

Fig. 4 High-resolution images of the BNs by (a) sonication and (b) hydrothermal reaction.
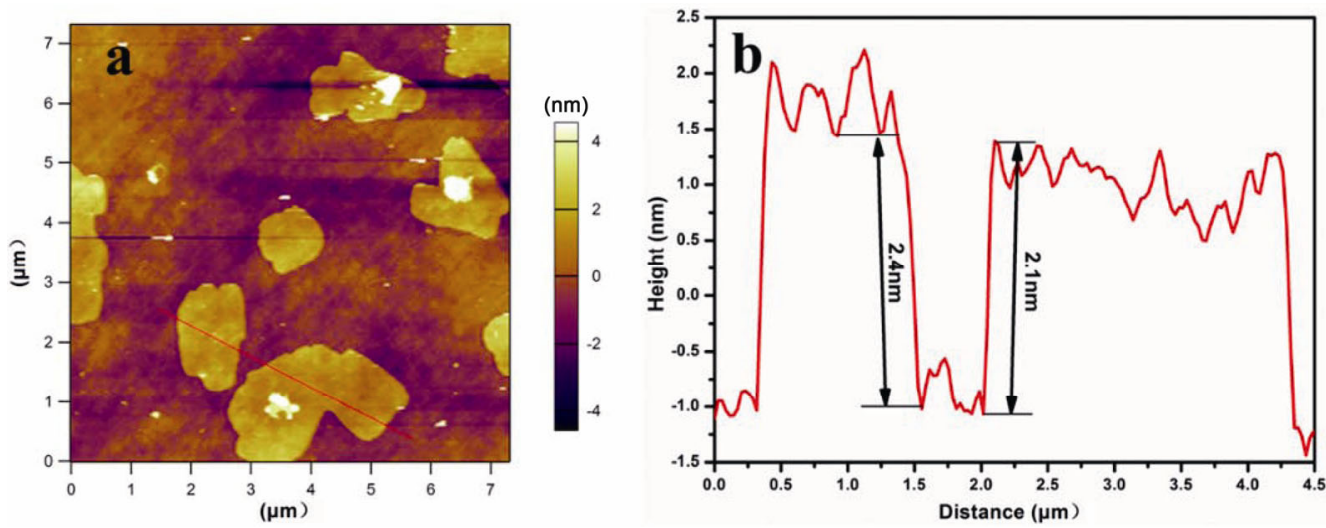

Fig. 5 (a) Tapping mode AFM image and (b) corresponding height profile of the SH-BNNS.

$1 \mathrm{~nm}$ owing to the trapped solvent between the BNNS and the underlying substrate [19]. Thus, the number of layers in the AFM data is less than 6, which is in good agreement with our HRTEM shown in Fig. 3(b). The AFM image also shows that the lateral size of the SH-BNNSs is largely more than $1 \mu \mathrm{m}$. 
As shown in Fig. 6, the BNNS with the treatment of sonication exhibits the thickness of $4.8 \mathrm{~nm}$, nearly 13 layers, while it treated by the hydrothermal is only $2.8 \mathrm{~nm}$, about 5 layers, with the lateral size of nearly 1 $\mu \mathrm{m}$. Interestingly, comparing Fig. 6(b) with Fig. 5(b) and Fig. 6(d), it is obvious that the rangeability of the HT-BNNS and SH-BN on the profiles is larger than the $\mathrm{SC}-\mathrm{BN}$ and the distribution is nonuniformity. It is possible that the solvent permeated into the layers under the high pressure produced in the process of hydrothermal, making some bubbles on the surface, which is corresbonding with our envisage.

Raman characterization is performed for the BNNS on the $\mathrm{Si} / \mathrm{SiO}_{2}$ substrate at the consistent condition (633 nm excitation source, $5 \mathrm{~s}$ for per cycle, 3 cycles). In Fig. 6, Raman spectra of SC-BNNS, HT-BNNS, and SH-BNNS show a dominant peak in the range of 1367.4-1369.6 $\mathrm{cm}^{-1}$, which is assigned to the highfrequency E2g mode (typically, single-crystal BN 1366 $\mathrm{cm}^{-1}$; highly ordered pyrolytic BN 1366-1367 $\mathrm{cm}^{-1}$; polycrystalline $\mathrm{BN}$ 1367-1374 $\mathrm{cm}^{-1}$ ) [20]. The intensity and full width at half maximum (FWHM) of the Raman peaks can be used to evaluate the crystallinity of h-BN [20-22]. Compared with the spectrum of BNNS, treated with different methods, the peak intensities of the HT-BNNS and SH-BNNS are much stronger than it treated with the sonication, indicating that the crystalline is much higher. The FWHM of the SC-BNNS is $12.69 \mathrm{~cm}^{-1}$, a little larger than the HT-BNNS $\left(11.31 \mathrm{~cm}^{-1}\right)$ and SH-BNNS $\left(11.62 \mathrm{~cm}^{-1}\right)$, which can be accounted for the lower crystallinity of the SC-BNNS. As the high energy of the sonication can make damage on the sheets of BNNS, at the atmosphere with high pressure and high temperature, it is not easy to destroy the lattice of the BNNS, keeping high crystallinity, which is in agreement with our TEM study, and again confirms our proposal. This suggests the exfoliating method of sonicationassisted hydrothermal is a more useful way to obtain high crystalline BNNS compared with conditional sonication treatment.

After having charactered the morphology and the crystallinity of BNNS, exfoliated by different methods, the yield was also determined and the details are as follows. The dispersions were transferred to a tube and centrifuged at $3500 \mathrm{rpm}$ for $20 \mathrm{~min}$. After centrifugation, the top $80 \mathrm{~mL}$ supernatant (out of $100 \mathrm{~mL}$ ) was carefully collected. The dispersion was allowed to settle for one
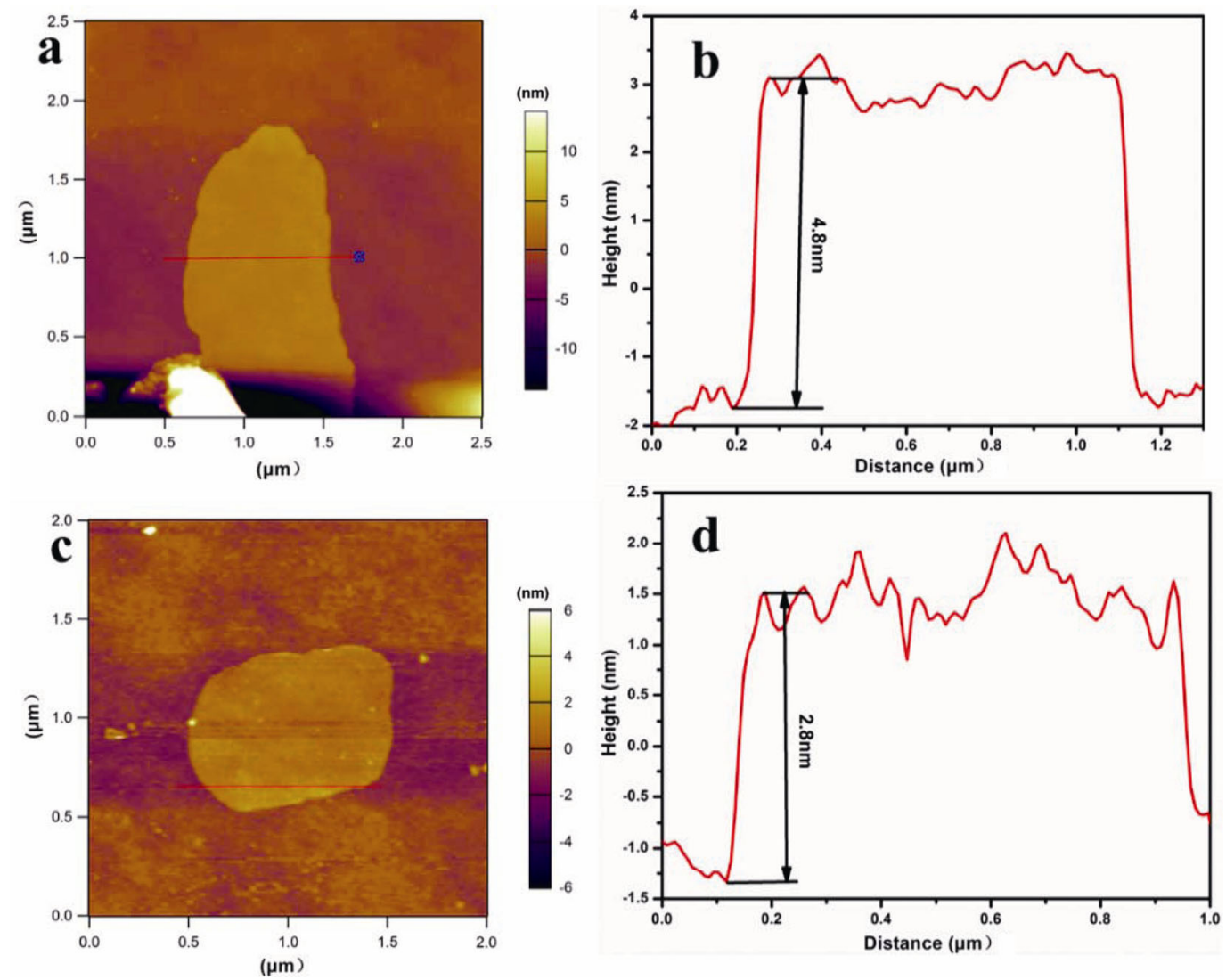

Fig. 6 Tapping mode AFM images and corresponding height profiles of the BNNS: (a, b) SC-BN, (c, d) HT-BN, respectively. 


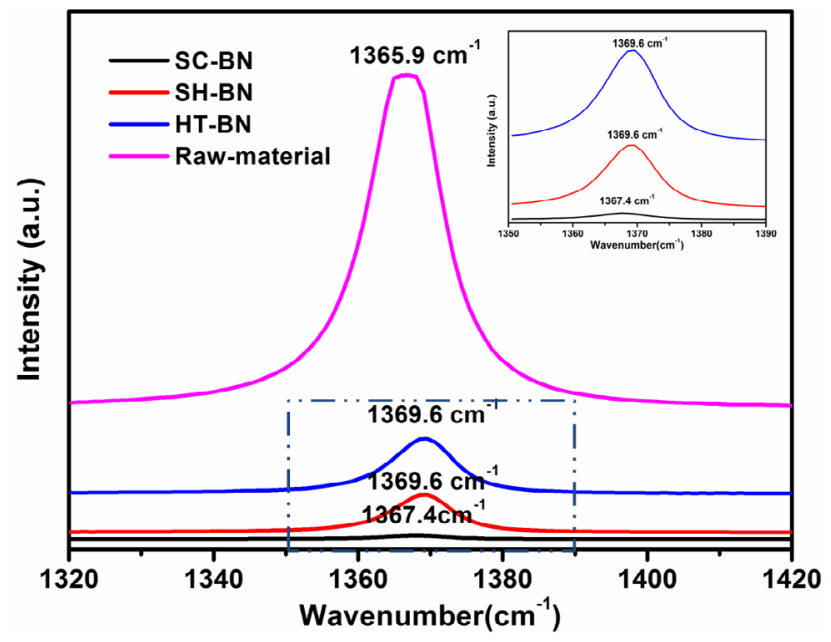

Fig. 7 Raman spectra of exfoliated BNNS by different methods.

month to subside out these undispersed particles as much as possible. The supernatant was carefully collected in a new vial with known mass and removed the solvents through freeze drying overnight. The mass of the BNNS can be determined by calculating the mass difference of the vial containing solid-state BNNS and the blank vial. Through counting, the corresponding yield is $0.88 \%$ of BNNS treated by sonication, $0.12 \%$ of HT-BNNS, and $1.68 \%$ of SH-BNNS, respectively, as shown in Fig. 8. It should be noted that a large amount of h-BN powders remained unreacted, which can be attributed to the inhomogeneous nature of solidstate reactions. The SEM images of the as-obtained products before rinsing provide direct support. These unreacted materials can be collected for subsequent exfoliation.

Based on the above analysis, it is obvious that sonication-assisted hydrothermal exfoliation is an efficient way to get high-crystallity BNNSs with large

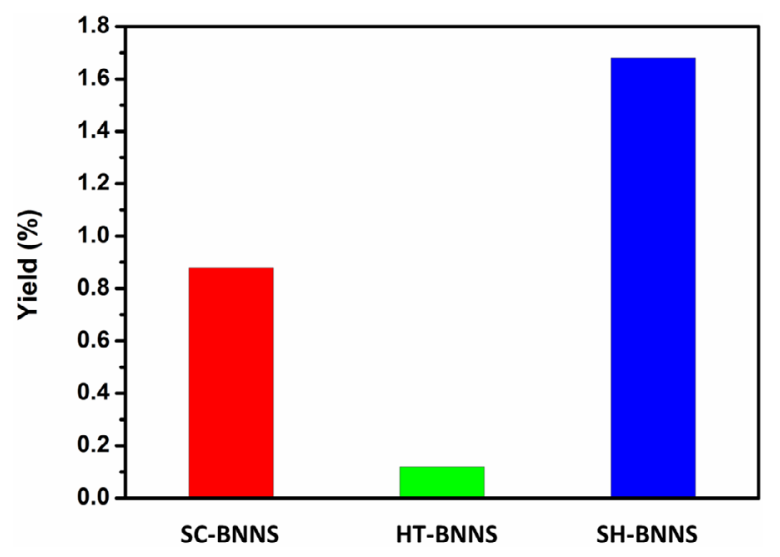

Fig. 8 Yields of different methods. yield. Here, we propose the key factors in the process, compared with traditional sonication and hydrothermal, respectively as follows. (1) Immerse the bulk BN into the $60 \%$ tertiary-butanol solution. As many researchers pointed out the optimal solvents are those with surface energy close to that of h-BN $[23,24]$. Herein, some research pointed out that the $60 \%$ tertiary-butanol solution is the best candidate to exfoliate and disperse h-BN, compared with other solution. Meanwhile, the melting boiling point is lower, and can evaporate at the bath temperature, producing high pressure under the bath temperature. (2) The tertiary-butanol and water enter into the interlayer space from the edges or defects with the assistance of sonication and the pressure. Researchers have revealed that the van der Waals force between the two water molecules is decreased with increasing temperature in a condition of high temperature and high pressure. This indicates the water molecules are force-free and have vigorous Brownian motion of molecules under sonication-assisted hydrothermal conditions [25-28]. Interestingly, $\mathrm{H}_{2} \mathrm{O}$ is a polar molecule of a V-shaped structure and the surface tensions of water are close to h-BN in hydrothermal conditions, suggesting that water molecules can be adsorbed on BNNSs, and separate the flakes. What is more, at the bath temperature of $355 \mathrm{~K}$, the interaction energy is calculated to be $-8.6 \mathrm{kcal} / \mathrm{mol}$, compared with -10.6 $\mathrm{kcal} / \mathrm{mol}$ at $297 \mathrm{~K} \mathrm{[29]}$. The sonication futherly weaks the interlayer bonding and reinforces the vigorous Brownian motion of molecules, improving more solution into the layers under the high pressure. (3) The cavitation effect of sonication acted on the $\mathrm{H}_{2} \mathrm{O}$ facilitates exfoliation of h-BN sheets from the surface of h-BN powders [30,31]. During the sonication, shear forces and caviation, i.e., the growth and collapse of the micrometer-sized bubbles or volid in the liquids due to pressure fluctuations, act on the inter-sheet attractive forces. Exfoliated BNs are dispersed by the 60\% tertiary-butanol solution, avoiding the stack of layers.

In summary, we have demonstrated a novel method to produce BNNS via sonication-assisted hydrothermal treatment, different from traditional sonication or hydrothermal method. Through combinating of the hydrothermal treatment and the sonication by one step, the high-quality BNNSs are prepared with a high yield. The exfoliation mechanism is put forward and reveals that the exfoliating process can be applicable to other two-dimensional materials. 


\section{References}

[1] Tan C, Cao X, Wu X-J, et al. Recent advances in ultrathin two-dimensional nanomaterials. Chem Rev 2017, 117: 6225-6331.

[2] Xue Y, Zhang Q, Wang W, et al. Opening two-dimensional materials for energy conversion and storage: A concept. Adv Energy Mater 2017, 7: 1602684.

[3] Voiry D, Yang J, Kupferberg J, et al. High-quality graphene via microwave reduction of solution-exfoliated graphene oxide. Science 2016, 353: 1413-1416.

[4] Zheng Z, Cox M, Li B. Surface modification of hexagonal boron nitride nanomaterials: A review. J Mater Sci 2018, 53: 66-99.

[5] Li P, Shen H, Qian Z, et al. Facile fabrication of flexible layered GO/BNNS composite films with high thermal conductivity. J Mater Sci 2018, 53: 4189-4198

[6] Lin Y, Connell JW. Advances in 2D boron nitride nanostructures: Nanosheets, nanoribbons, nanomeshes, and hybrids with graphene. Nanoscale 2012, 4: 6908-6939.

[7] Li Q, Chen L, Gadinski MR, et al. Flexible hightemperature dielectric materials from polymer nanocomposites. Nature 2015, 523: 576-579.

[8] Lee D, Song SH. Ultra-thin ultraviolet cathodoluminescent device based on exfoliated hexagonal boron nitride. RSC $A d v$ 2017, 7: 7831-7835.

[9] Marconnet AM, Yamamoto N, Panzer MA, et al. Thermal conduction in aligned carbon nanotube-polymer nanocomposites with high packing density. ACS Nano 2011, 5: 4818-4825.

[10] Xiao F, Naficy S, Casillas G, et al. Hydrogels: Edgehydroxylated boron nitride nanosheets as an effective additive to improve the thermal response of hydrogels. $A d v$ Mater 2015, 27: 7247.

[11] Liu Z, Gong Y, Zhou W, et al. Ultrathin high-temperature oxidation-resistant coatings of hexagonal boron nitride. Nat Commun 2013, 4: 2541-2546.

[12] Lee D, Lee B, Park KH, et al. Scalable exfoliation process for highly soluble boron nitride nanoplatelets by hydroxideassisted ball milling. Nano Lett 2015, 15: 1238-1244.

[13] Li X, Hao X, Zhao M, et al. Exfoliation of hexagonal boron nitride by molten hydroxides. Adv Mater 2013, 25: 2200-2204.

[14] Rafiei-Sarmazdeh Z, Jafari SH, Ahmadi SJ, et al. Largescale exfoliation of hexagonal boron nitride with combined fast quenching and liquid exfoliation strategies. J Mater Sci 2016, 51: 3162-3169.

[15] Liu L, Park J, Siegel DA, et al. Heteroepitaxial growth of two-dimensional hexagonal boron nitride templated by graphene edges. Science 2014, 343: 163-167.

[16] Sajjad M, Ahmadi M, Guinel MJ-F, et al. Large scale synthesis of single-crystal and polycrystalline boron nitride nanosheets. J Mater Sci 2013, 48: 2543-2549.

[17] Lotya M, Hernandez Y, King PJ, et al. Liquid phase production of graphene by exfoliation of graphite in surfactant/water solutions. J Am Chem Soc 2009, 131: 3611-3620.

[18] Du M, Wu Y, Hao X. A facile chemical exfoliation method to obtain large size boron nitride nanosheets. CrystEngComm
2013, 15: 1782-1786.

[19] Coleman JN, Lotya M, O'Neill A, et al. Two-dimensional nanosheets produced by liquid exfoliation of layered materials. Science 2011, 331: 568-571.

[20] Gorbachev RV, Riaz I, Nair RR, et al. Hunting for monolayer boron nitride: Optical and Raman signatures. Small 2011, 7: 465-468.

[21] Guo H, Gao J, Ishida N, et al. Characterization of twodimensional hexagonal boron nitride using scanning electron and scanning helium ion microscopy. Appl Phys Lett 2014, 104: 031607.

[22] Nemanich RJ, Solin SA, Martin RM. Light scattering study of boron nitride microcrystals. Phys Rev B 1981, 23: 6348-6354.

[23] Li C, Wang T, Wu Y, et al. Fabrication of two-dimensional nanosheets via water freezing expansion exfoliation. Nanotechnology 2014, 25: 495302.

[24] Lee KH, Shin H-J, Lee J, et al. Large-scale synthesis of high-quality hexagonal boron nitride nanosheets for large-area graphene electronics. Nano Lett 2012, 12: 714-718.

[25] Liu X, Zheng M, Xiao K, et al. Simple, green and high-yield production of single- or few-layer graphene by hydrothermal exfoliation of graphite. Nanoscale 2014, 6: 4598-4603.

[26] Modell M. Processing methods for the oxidation of organics in supercritical water. U.S. Patent 4,338,199. 1982.

[27] Byrappa K, Adschiri T. Hydrothermal technology for nanotechnology. Prog Cryst Growth Ch 2007, 53: 117-166.

[28] Byrappa K, Yoshimura M. Handbook of Hydrothermal Technology. William Andrew, 2012.

[29] Zhu W, Gao X, Li Q, et al. Controlled gas exfoliation of boron nitride into few-layered nanosheets. Angew Chem Int Ed 2016, 55: 10766-10770.

[30] Lin Y, Williams TV, Xu T-B, et al. Aqueous dispersions of few-layered and monolayered hexagonal boron nitride nanosheets from sonication-assisted hydrolysis: Critical role of water. J Phys Chem C 2011, 115: 2679-2685.

[31] Mason TJ, Lorimer JP. Applied Sonochemistry: The Uses of Power Ultrasound in Chemistry and Processing. WileyVCH Verlag, Weinheim, 2002.

Open Access This article is licensed under a Creative Commons Attribution 4.0 International License, which permits use, sharing, adaptation, distribution and reproduction in any medium or format, as long as you give appropriate credit to the original author(s) and the source, provide a link to the Creative Commons licence, and indicate if changes were made.

The images or other third party material in this article are included in the article's Creative Commons licence, unless indicated otherwise in a credit line to the material. If material is not included in the article's Creative Commons licence and your intended use is not permitted by statutory regulation or exceeds the permitted use, you will need to obtain permission directly from the copyright holder.

To view a copy of this licence, visit http://creativecommons. org/licenses/by/4.0/. 